\begin{tabular}{|c|l|}
\hline Title & Projections of surfaces in the hyperbolic space al ong horocy cles \\
\hline Author(s) & Izumiya, Shyuichi; Tari, Farid \\
\hline Citation & Hokkaido University Preprint Series in Mathematics, 887, 1-22 \\
\hline Issue Date & 2008-01-09 \\
\hline DOI & 10.14943/84037 \\
\hline Doc URL & http://hdl.handle.net/2115/69696 \\
\hline Type & bulletin (article) \\
\hline File Information & pre887.pdf \\
\hline
\end{tabular}

Instructions for use 


\title{
Projections of surfaces in the hyperbolic space along horocycles
}

\author{
Shyuichi Izumiya and Farid Tari
}

January 9, 2008

\begin{abstract}
We study in this paper orthogonal projections of embedded surfaces $M$ in $H_{+}^{3}(-1)$ along horocycles to planes. The singularities of the projections capture the extrinsic geometry of $M$ related to the lightcone Gauss map. We give geometric characterisations of these singularities and prove a Koenderink type theorem which relates the hyperbolic curvature of the surface to the curvature of the profile and of the normal section of the surface. We also prove duality results concerning the bifurcation set of the family of projections.
\end{abstract}

\section{Introduction}

The work of this paper is part of a wider project of investigating the extrinsic geometry of submanifolds embedded in the pseudo-spheres in the Minkowski space $\mathbb{R}_{1}^{n}$ via singularity theory. The extrinsic geometric information is obtained by considering the contact of the submanifold with degenerate objects in the given pseudo-sphere. By degenerate we mean, for instance, a flat object (that is, a submanifold with some curvature vanishing everywhere).

We studied in [27] the contact of surfaces in the hyperbolic space with geodesics. This is measured by the singularities of orthogonal projections of the surface along geodesics to hyperplanes and horospheres. The expression of an orthogonal projection along a geodesic is also given in [28] using a different method. The work in [27] is analogous to that on orthogonal projections of surfaces in the Euclidean and projective 3 -spaces (these are well studied; see for example [1, 3, 4, 6, 7, 9, 11, 29, 30, 32, 33, 34,

2000 Mathematics Subject classification 53A35, 58K05.

Key Words and Phrases. Bifurcation sets, contours, Legendrian duality, projections, profiles, hyperbolic space, singularities, de Sitter space, lightcone. 
35]). Analogous in [27] means that we view a line in the Euclidean space as a geodesic. But a line in the Euclidean space can also be viewed as the limit of circles with radii tending to infinity. In the hyperbolic space, the limit of circles with radii tending to infinity is a horocycle. So it is natural to consider, as we do in this paper, projections in the hyperbolic space along horocycles.

Another reason we consider projections along horocycles is the following. Given $p \in M$, there is a well defined unit normal vector $e(p)$ to $M$ at $p$; see $\S 2$. (If $M$ is orientable, then $e(p)$ is globally defined. However, it is always locally defined and our investigation here is local in nature.) The vector $e(p)$ is in the de Sitter space $S_{1}^{3}$ and we have the de Sitter Gauss map

$$
\begin{aligned}
\mathbb{E}: M & \rightarrow S_{1}^{3} \\
p & \rightarrow e(p)
\end{aligned}
$$

The projections of $M$ along geodesics pick up extrinsic geometric information about $M$ related to the de Sitter Gauss map, see [27]. For example, the projection along a geodesic has a cusp singularity at $p$ if and only if the tangent to the geodesic at $p$ is a de Sitter asymptotic direction. The points on $M$ where the projection has a swallowtail singularity is precisely the locus of points of geodesic inflections of the de Sitter asymptotic curves. Also, the projection has a lips/beaks singularity at $p$ if and only if $p$ is a de Sitter parabolic point and the tangent to the geodesic is along the unique de Sitter asymptotic direction at $p$.

There is another Gauss map on the surface introduced in [19] and called the lightcone Gauss map; see $\S 2$. The vector $p \pm e(p)$ is lightlike (i.e., belongs to the lighcone $\left.L C^{*}\right)$, so we have the lightcone Gauss maps

$$
\begin{aligned}
\mathbb{L}^{ \pm}: M & \rightarrow L C^{*} \\
p & \rightarrow p \pm e(p)
\end{aligned}
$$

Projecting along horocycles is a natural candidate to pick up extrinsic geometric information about the surface related to the lightcone Gauss map. We expect, for instance, the projection along a horocycle with tangent at $p$ along a horo-asymptotic direction (i.e., a direction $u$ satisfying $\left\langle d_{p}\left(\mathbb{L}^{ \pm}\right)(u), u\right\rangle=0$ ) to have a cusp singularity at $p$.

We give in $\S 3$ the expression of the family $P$ of projections along horocycles to orthogonal planes. The planes of projection are arbitrary, that is, projecting to parallel orthogonal planes yields the same information. So we project to the planes that passe through the point $p_{0}=(1,0,0,0)$. Then the horocycles of interest are determined by a pair of vectors $(l, v)$ in the set $\mathcal{C}=\left\{(l, v) \in S_{+}^{2} \times S_{0}^{2} \mid\langle l, v\rangle=0\right\}$, where $S_{+}^{2}$ and $S_{0}^{2}$ are spheres in $L C^{*}$ and $S_{1}^{3}$ respectively. The set $\mathcal{C}$ is in fact the parameter space of our family of projections. For $(l, v)$ fixed, the map $P_{(l, v)}$ can be considered locally as a map-germ from $\mathbb{R}^{2}, 0 \rightarrow \mathbb{R}^{2}, 0$. We show in $\S 4$ that $P_{(l, v)}$ has a cusp singularity or worse if and only if $\kappa\left(v^{*}\right)=-\langle l, e(p)\rangle /\langle l, p\rangle$, where $v^{*}$ is the tangent to the horocycle $(l, v)$ 
at $p$ and $\kappa\left(v^{*}\right)$ is the de Sitter normal curvature at $p$ along $v^{*}$. The above relation yields several interesting geometric properties of $M$ (Theorem 4.2). For instance, $\kappa\left(v^{*}\right)= \pm 1$ if and only if $v^{*}$ is a horo-asymptotic direction and $l=\widetilde{\mathbb{L}^{ \pm}}(p)$, where $\widetilde{\mathbb{L}^{ \pm}}(p)$ is the radial projection of $\mathbb{L}^{ \pm}(p)$ to $S_{+}^{2}$. We also show that the points $p \in M$ where the projection along the horocycle $\left(\widetilde{\mathbb{L}^{ \pm}}(p), v\right)$ has a swallowtail singularity at $p$ is precisely the locus of geodesic inflections of the horo-asymptotic curves (Proposition 4.3). We then prove a Koenderink type theorem which relates the hyperbolic curvature of the surface to the curvature of the profile and of the normal section of the surface (Theorem 4.4).

We prove in $\S 5$ a duality result between some surfaces associated to $M$ and some special curves on the $\Delta_{2}$-dual surface $M^{(2, *)}$ of $M$; (see $\S 6$ ). The result is analogous to that of Shcherback in [34]. We also prove a duality result concerning the bifurcation sets of the families of projections, analogous to that of Bruce-Romero Fuster in [9]. In $\S 5$, we use the duality concepts introduced in $[13,14]$; see $\S 6$ for details.

\section{Preliminaries}

The Minkowski space $\left(\mathbb{R}_{1}^{4},\langle\rangle,\right)$ is the 4 -dimensional vector space $\mathbb{R}^{4}$ endowed with the pseudo scalar product $\langle x, y\rangle=-x_{0} y_{0}+\sum_{i=1}^{3} x_{i} y_{i}$, where $x=\left(x_{0}, x_{1}, x_{2}, x_{3}\right)$ and $y=\left(y_{0}, x_{1}, x_{2}, y_{3}\right)$ in $\mathbb{R}_{1}^{4}$. We say that a vector $x$ in $\mathbb{R}_{1}^{4} \backslash\{0\}$ is spacelike, lightlike or timelike if $\langle x, x\rangle>0,=0$ or $<0$ respectively. The norm of a vector $x \in \mathbb{R}_{1}^{4}$ is defined by $\|x\|=\sqrt{|\langle x, x\rangle|}$.

Given a vector $v \in \mathbb{R}_{1}^{4}$ and a real number $c$, the hyperplane with pseudo normal $v$ is defined by

$$
H P(v, c)=\left\{x \in \mathbb{R}_{1}^{4} \mid\langle x, v\rangle=c\right\} .
$$

We say that $\operatorname{HP}(v, c)$ is a spacelike, timelike or lightlike hyperplane if $v$ is timelike, spacelike or lightlike respectively. We have the following three types of pseudo-spheres in $\mathbb{R}_{1}^{4}$ :

$$
\begin{array}{rll}
\text { Hyperbolic 3-space : } & H^{3}(-1) & =\left\{x \in \mathbb{R}_{1}^{4} \mid\langle x, x\rangle=-1\right\}, \\
\text { de Sitter } 3 \text {-space : } & S_{1}^{3} & =\left\{x \in \mathbb{R}_{1}^{4} \mid\langle x, x\rangle=1\right\}, \\
\text { (open) lightcone : } & L C^{*} & =\left\{x \in \mathbb{R}_{1}^{4} \backslash\{0\} \mid\langle x, x\rangle=0\right\} .
\end{array}
$$

We also define the lightcone sphere

$$
S_{+}^{2}=\left\{x \in L C^{*} \mid\langle x, x\rangle=0, x_{0}=1\right\}
$$

and the Euclidean sphere

$$
S_{0}^{2}=\left\{x \in S_{1}^{3} \mid\langle x, x\rangle=1, x_{0}=0\right\} .
$$

For $x \in L C^{*}$, we have $x_{0} \neq 0$ so

$$
\widetilde{x}=\left(1, \frac{x_{1}}{x_{0}}, \frac{x_{2}}{x_{0}}, \frac{x_{3}}{x_{0}}\right) \in S_{+}^{2} .
$$


The hyperbolic space has two connected components. We only consider embedded surfaces in $H_{+}^{3}(-1)=\left\{x \in H^{3}(-1) \mid x_{0} \geq 1\right\}$ as the study is similar for those embedded in $H_{-}^{3}(-1)=\left\{x \in H^{3}(-1) \mid x_{0} \leq-1\right\}$.

The wedge product of 4 vectors $a_{1}, a_{2}, a_{3} \in \mathbb{R}_{1}^{4}$ is given by

$$
a_{1} \wedge a_{2} \wedge a_{3}=\left|\begin{array}{cccc}
-e_{0} & e_{1} & e_{2} & e_{3} \\
a_{0}^{1} & a_{1}^{1} & a_{2}^{1} & a_{3}^{1} \\
a_{0}^{2} & a_{1}^{2} & a_{2}^{2} & a_{3}^{2} \\
a_{0}^{3} & a_{1}^{3} & a_{2}^{3} & a_{3}^{3}
\end{array}\right|
$$

where $\left\{e_{0}, e_{1}, e_{2}, e_{3}\right\}$ is the canonical basis of $\mathbb{R}_{1}^{4}$ and $a_{i}=\left(a_{0}^{i}, a_{1}^{i}, a_{2}^{i}, a_{3}^{i}\right), i=1,2,3$. One can check that $\left\langle a, a_{1} \wedge a_{2} \wedge a_{3}\right\rangle=\operatorname{det}\left(a, a_{1}, a_{2}, a_{3}\right)$, so the vector $a_{1} \wedge a_{2} \wedge a_{3}$ is pseudo orthogonal to all the vectors $a_{i}, i=1,2,3$.

Some aspects of the extrinsic geometry of hypersurfaces in the hyperbolic space are studied in [13]-[23] and [26, 27]. Let $M$ be a surface embedded in $H_{+}^{3}(-1)$. Given a local chart $\boldsymbol{i}: U \rightarrow M$, where $U$ is an open subset of $\mathbb{R}^{2}$, we denote by $\boldsymbol{x}: U \rightarrow H_{+}^{3}(-1)$ such embedding, identify $\boldsymbol{x}(U)$ with $U$ through the embedding $\boldsymbol{x}$ and write $M=\boldsymbol{x}(U)$. Since $\langle\boldsymbol{x}, \boldsymbol{x}\rangle \equiv-1$, we have $\left\langle\boldsymbol{x}_{u_{i}}, \boldsymbol{x}\right\rangle \equiv 0$, for $i=1,2$, where $u=\left(u_{1}, u_{2}\right) \in U$. We define the spacelike unit normal vector $e(u)$ to $M$ at $\boldsymbol{x}(u)$ by

$$
e(u)=\frac{\boldsymbol{x}(u) \wedge \boldsymbol{x}_{u_{1}}(u) \wedge \boldsymbol{x}_{u_{2}}(u)}{\left\|\boldsymbol{x}(u) \wedge \boldsymbol{x}_{u_{1}}(u) \wedge \boldsymbol{x}_{u_{2}}(u)\right\|}
$$

It follows that the vector $\boldsymbol{x} \pm e$ is a lightlike vector. Let

$$
\mathbb{E}: U \rightarrow S_{1}^{n} \quad \text { and } \quad \mathbb{L}^{ \pm}: U \rightarrow L C^{*}
$$

be the maps defined by $\mathbb{E}(u)=e(u)$ and $\mathbb{L}^{ \pm}(u)=\boldsymbol{x}(u) \pm e(u)$. These are called, respectively, the de Sitter Gauss map and the lightcone Gauss map (or hyperbolic Gauss indicatrix) of $M$ ([19]). For any $p=\boldsymbol{x}\left(u_{0}\right) \in M$ and $v \in T_{p} M$, one can show that $D_{v} \mathbb{E} \in T_{p} M$, where $D_{v}$ denotes the covariant derivative with respect to the tangent vector $v$. Since the derivative $d \boldsymbol{x}\left(u_{0}\right)$ can be identified with the identity mapping $1_{T_{p} M}$ on the tangent space $T_{p} M$, we have $d \mathbb{L}^{ \pm}\left(u_{0}\right)=1_{T_{p} M} \pm d \mathbb{E}\left(u_{0}\right)$, under the identification of $U$ and $M$ via the embedding $\boldsymbol{x}$.

The linear transformation $A_{p}=-d \mathbb{E}\left(u_{0}\right)$ is called the de Sitter shape operator. Its eigenvalues $\kappa_{i}, i=1,2$, are called the de Sitter principal curvature and the corresponding eigenvectors $p_{i}, i=1,2$, are called the de Sitter principal directions.

The linear transformation $S_{p}^{ \pm}=-d \mathbb{L}^{ \pm}\left(u_{0}\right)$ is labelled the lightcone shape operator of $M$ at $p$. It has the same eigenvectors as $A_{p}$ but its eigenvalues are distinct from those of $A_{p}$. In fact the eigenvalues $\bar{\kappa}_{i}^{ \pm}$of $S_{p}^{ \pm}$satisfy $\bar{\kappa}_{i}^{ \pm}=-1 \pm \kappa_{i}, i=1,2$.

We call $K_{e}(p)=\operatorname{det}\left(A_{p}\right)=\kappa_{1}(p) \kappa_{2}(p)\left(\operatorname{resp} . K_{h}(p)=\operatorname{det}\left(S_{p}^{ \pm}\right)=\bar{\kappa}_{1}(p) \overline{\kappa_{2}}(p)\right)$ the de Sitter (resp. hyperbolic) Gauss-Kronecker curvature of $M$ at $p$. The curvature $K_{e}$ is also called the extrinsic Gaussian curvature. The set of points where $K_{e}(p)=0$ (resp. 
$K_{h}^{ \pm}(p)=0$ ) is labelled the de Sitter (resp. horospherical, horo- for short) parabolic set of $M$. The restriction of the pseudo-scalar product to the hyperbolic space is a scalar product, so $H_{+}^{3}(-1)$ is a Rimaniann manifold. We have the sectional curvature $K_{I}$ of $M$ which is also called the intrinsic Gaussian curvature. It is known that $K_{e}=K_{I}+1$ (see $§ 2.2$ in $[10]$ ).

The operators $A_{p}$ and $S_{p}^{ \pm}$are self-adjoint operators on $M$, so we can define the notion of asymptotic directions at $p$. We say that $u \in T_{p} M$ is a de Sitter (resp. horo-) asymptotic direction if and only if $\left\langle A_{p}(u), u\right\rangle=0\left(\operatorname{resp} .\left\langle S_{p}^{ \pm}(u), u\right\rangle=0\right)$. There are $0 / 1 / 2$ de Sitter (resp. horo-) asymptotic directions at every point where $K_{e}(p)$ (resp. $\left.K_{h}(p)\right) 0>/=/<0$.

We also define the de Sitter normal curvature at $p$ along a direction $u \in T_{p} M$ to be $\kappa(u)=\left\langle A_{p}(u), u\right\rangle /\langle u, u\rangle$. The horo-normal curvature at $p$ along a direction $u \in T_{p} M$ is defined similarly and is given by $\kappa_{h}^{ \pm}(u)=\left\langle S_{p}^{ \pm}(u), u\right\rangle /\langle u, u\rangle$.

A surface given by the intersection of $H_{+}^{3}(-1)$ with a spacelike, timelike or lightlike hyperplane is called respectively sphere, equidistant surface or horosphere. The intersection of the surface with timelike hyperplane through the origin is called simply a plane. Planes are the only surfaces with everywhere zero de Sitter Gaussian curvature ([21]). Horospheres are the only surfaces with everywhere zero hyperbolic curvature $([21])$.

\section{The family of projections along horocycles}

We need some preliminaries about curves in $H_{+}^{3}(-1)$. Let $\gamma: I \rightarrow H_{+}^{3}(-1)$ be a regular curve. Since $H_{+}^{3}(-1)$ is a Riemannian manifold, we can parametrise $\gamma$ by arc-length and assume that $\gamma(s)$ is unit speed. Let $t(s)=\gamma^{\prime}(s)$, with $\|t(s)\|=1$. The vector $t^{\prime}(s)$ is not in the tangent space $T_{\gamma(s)} H_{+}^{3}(-1)$, so we project it along $\gamma$ to this tangent space. The resulting vector is $t^{\prime}(s)-\gamma(s)$. Now if $\left\langle t^{\prime}(s), t^{\prime}(s)\right\rangle \neq-1$, then $\left\|t^{\prime}(s)-\gamma(s)\right\| \neq 0$. In this case, we define the unit normal vector to the curve as the vector $n(s)=\frac{t^{\prime}(s)-\gamma(s)}{\left\|t^{\prime}(s)-\gamma(s)\right\|}$. If $e(s)=\gamma(s) \wedge t(s) \wedge n(s)$, then we have a pseudo orthogonal frame $\{\gamma(s), t(s), n(s), e(s)\}$ in $\mathbb{R}_{1}^{4}$ along $\gamma$. Frenet-Serret type formulae, similar to those for a space curve in $\mathbb{R}^{3}$, can be proved for the curve $\gamma([22])$ and are as follows

$$
\left\{\begin{aligned}
\gamma^{\prime}(s) & =t(s) \\
t^{\prime}(s) & =\gamma(s)+\kappa_{h}(s) n(s) \\
n^{\prime}(s) & =-\kappa_{h}(s) t(s)+\tau_{h}(s) e(s) \\
e^{\prime}(s) & =-\tau_{h}(s) n(s)
\end{aligned}\right.
$$

where $\kappa_{h}(s)=\left\|t^{\prime}(s)-\gamma(s)\right\|$ and $\tau_{h}(s)=-\operatorname{det}\left(\gamma(s), \gamma^{\prime}(s), \gamma^{\prime \prime}(s), \gamma^{\prime \prime \prime}(s)\right) / \kappa_{h}(s)^{2}$. The quantities $\kappa_{h}(s)$ and $\tau_{h}(s)$ are called the curvature (resp. torsion) of the curve $\gamma$ at $\gamma(s)$. The condition $\left\langle t^{\prime}(s), t^{\prime}(s)\right\rangle \neq-1$ above is in fact equivalent to $\kappa_{h}(s) \neq 0$. (See 
[22] for more results on curves in the hyperbolic plane.)

When the curve $\gamma$ is not parametrised by arc length, the formula for the curvature is

$$
\kappa_{h}(\theta)=\left\|\frac{1}{l^{\prime}(\theta)^{3}}\left(\gamma^{\prime \prime}(\theta) l^{\prime}(\theta)-\gamma^{\prime}(\theta) l^{\prime \prime}(\theta)\right)-\gamma(\theta)\right\|,
$$

where

$$
l(\theta)=\int_{0}^{\theta}\left\|\gamma^{\prime}(\alpha)\right\| d \alpha .
$$

A horocycle in $H_{+}^{3}(-1)$ is the intersection of a horosphere with a plane orthogonal to the horosphere. Let $H S^{3}(l, c)=H_{+}^{3}(-1) \cap H P(l, c)$, with $l \in S_{+}^{2} \subset L C^{*}$ and $c \in \mathbb{R}$, denote a horosphere in $H_{+}^{3}(-1)$. Then a horocycle is given by $H S^{3}(l, c) \cap H P(v, 0)$, with $v \in S_{1}^{3}$ and $\langle l, v\rangle=0$. Fixing $l$ and $v$ and varying $c$ gives parallel horocycles.

A smooth curve in $H_{+}^{3}(-1)$ is a horocycle if and only if $\kappa_{h} \equiv 1$ and $\tau_{h} \equiv 0$ ([18]). Also, for a curve in a hyperbolic plane (which is the case of a horocycle), $\kappa_{h}=\kappa_{g}$, where $\kappa_{g}$ denotes its geodesic curvature, and the hyperbolic curvature $K_{h}=\kappa_{g}-1=\kappa_{h}-1$. Therefore, for a horocycle we have $K_{h} \equiv 0$, which means that they are flat objects.

We are considering here orthogonal projections along horocycles to planes. Projecting to two parallel planes yields the same information, so we choose those that pass through the point $p_{0}=(1,0,0,0)$. These are parametrised by the sphere $S_{0}^{2}$. So parallel horocycles in $H_{+}^{3}(-1)$ that are of interest are those determined by the elements of the set

$$
\mathcal{C}=\left\{(l, v) \in S_{+}^{2} \times S_{0}^{2} \mid\langle l, v\rangle=0\right\} .
$$

Given a plane $H P(v, 0) \cap H_{+}^{3}(-1)$, with $v \in S_{0}^{2}$, and a point $p \in H_{+}^{3}(-1)$, there is a unique horocycle through $p$, determined by a unique $(l, v) \in \mathcal{C}$, which intersects orthogonally the given plane at some point $q(p,(l, v))$. We call the point $q(p,(l, v))$ the orthogonal projection of $p$ along the horocycle $(l, v)$.

Let $\pi: \mathcal{C} \rightarrow S_{0}^{2}$ be the projection $\pi(l, v)=v$ and $i: S_{0}^{2} \rightarrow S_{1}^{3}$ be the inclusion $i(v)=v$. Let

$$
\mathcal{F}=\left\{(v, w) \in T S_{1}^{3} \mid\langle w, w\rangle=-1\right\} \rightarrow S_{1}^{3}
$$

be the timelike unit spherical bundle over $S_{1}^{3}$.

Theorem 3.1 The family of projections along horocycles in $H_{+}^{3}(-1)$ is given by

$$
\begin{array}{rlc}
P: \quad H_{+}^{3}(-1) \times \mathcal{C} & \rightarrow & (i \circ \pi)^{*} \mathcal{F} \\
(p,(l, v)) & \mapsto \quad((l, v), q(p,(l, v)))
\end{array}
$$

where

$$
q(p,(l, v))=p-\langle p, v\rangle v+\frac{1}{2} \frac{\langle p, v\rangle^{2}}{\langle p, l\rangle} l .
$$


Proof. A parametrisation of the horocycle $(l, v)$ which is orthogonal to the plane $H P(v, 0) \cap H_{+}^{3}(-1)$ at $q(p,(l, v))$ is given by

$$
c(s)=q(p,(l, v))+s v+\frac{s^{2}}{2} \lambda l .
$$

for some scalar $\lambda$, see [24]. The horocycle $c(s)$ passes through $p$ if $p=q(p,(l, v))+$ $s_{0} v+\frac{s_{0}^{2}}{2} \lambda l$ for some $s_{0} \in \mathbb{R}$. So $q(p,(l, v))=p-s_{0} v-\frac{s_{0}^{2}}{2} \lambda l$. We have

$$
\langle q(p,(l, v)), v\rangle=\langle p, v\rangle-s_{0}=0,
$$

which gives $s_{0}=\langle p, v\rangle$. As $c\left(s_{0}\right) \in H_{+}^{3}(-1),\left\langle c\left(s_{0}\right), c\left(s_{0}\right)\right\rangle=-1$, we have

$$
\langle q(p,(l, v)), \lambda l\rangle=\langle p, \lambda l\rangle=-1,
$$

therefore $l=-1 /\langle p, l\rangle$. Hence $q(p,(l, v))=p-\langle p, v\rangle v+\frac{1}{2} \frac{\langle p, v\rangle^{2}}{\langle p, l\rangle} l$.

In this paper, the family of orthogonal projections of a given surface $M$ in $H_{+}^{3}(-1)$ along horocycles refers to the restriction of the family $P$ to $M$. We still denote this restriction by $P$. We have the following result where the term generic is defined in terms of transversality to submanifolds of multi-jet spaces (see for example [12]).

Theorem 3.2 For a residual set of embeddings $x: M \rightarrow H_{+}^{3}(-1)$, the family $P$ is a generic family of mappings.

Proof. The theorem follows from Montaldi's result in [31] and the fact that $P$ : $H_{+}^{3}(-1) \times \mathcal{C} \rightarrow(i \circ \pi)^{*} \mathcal{F}$ is a stable map.

We denote by $P_{(l, v)}$ the map $H_{+}^{3}(-1) \rightarrow H_{+}^{3}(-1)$, given by $P_{(l, v)}(p)=q(p,(l, v))$, with $q(p,(l, v))$ as in Theorem 3.1. We also keep the same notation for the restriction of $P_{(l, v)}$ to the surface $M \subset H_{+}^{3}(-1)$.

For a given $(l, v) \in \mathcal{C}$ and a point $p_{0} \in M$, one can choose local coordinates so that $P_{(l, v)}$ restricted to $M$ can be considered locally as a map-germ $\mathbb{R}^{2}, 0 \rightarrow \mathbb{R}^{2}, 0$. These map-germs are extensively studied. We refer to [32] for the list of the $\mathcal{A}$-orbits with $\mathcal{A}_{e}$-codimension $\leq 6$, where $\mathcal{A}$ denotes the Mather group of smooth changes of coordinates in the source and target. In Table 1, we reproduce from [32] the list of local singularities of $\mathcal{A}_{e}$-codimension $\leq 3$. Some of these singularities are also called as follows: $4_{2}$ (lips/beaks), $4_{3}$ (goose), 5 (swallowtail), 6 (butterfly), $11_{5}$ (gulls).

It follows from Theorem 3.2 that for generic embeddings of the surface only sin-

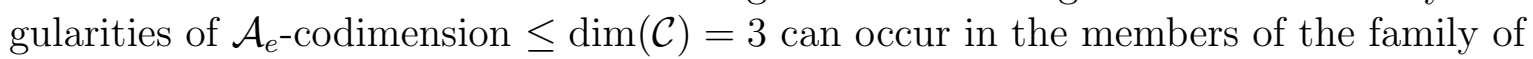
orthogonal projections. So we have the following result.

Proposition 3.3 For a residual set of embeddings $x: M \rightarrow H_{+}^{3}(-1)$, the projections $P_{(l, v)}: M \rightarrow H_{+}^{3}(-1)$ in the family $P$ have local singularities $\mathcal{A}$-equivalent to one in Table 1 whose $\mathcal{A}_{e}$-codimension $\leq 3$. Moreover, these singularities are versally unfolded by the family $P$. 
Table 1: $\mathcal{A}_{e}$-codimension $\leq 3$ local singularities of map-germs $\mathbb{R}^{2}, 0 \rightarrow \mathbb{R}^{2}, 0([32])$.

\begin{tabular}{llc}
\hline Name & Normal form & $\mathcal{A}_{e}$-codimension \\
\hline Immersion & $(x, y)$ & 0 \\
Fold & $\left(x, y^{2}\right)$ & 0 \\
Cusp & $\left(x, x y+y^{3}\right)$ & 0 \\
$4_{k}$ & $\left(x, y^{3} \pm x^{k} y\right), k=2,3,4$ & $k-1$ \\
5 & $\left(x, x y+y^{4}\right)$ & 1 \\
6 & $\left(x, x y+y^{5} \pm y^{7}\right)$ & 2 \\
7 & $\left(x, x y+y^{5}\right)$ & 3 \\
$11_{2 k+1}$ & $\left(x, x y^{2}+y^{4}+y^{2 k+1}\right), k=2,3$ & $k$ \\
12 & $\left(x, x y^{2}+y^{5}+y^{6}\right)$ & 3 \\
16 & $\left(x, x^{2} y+y^{4} \pm y^{5}\right)$ & 3 \\
\hline
\end{tabular}

The members of $P$ can also have multi-local local singularities with $\mathcal{A}_{e}$-codimension $\leq 3$, and these singularities are also versally unfolded by the family $P$. However, in this paper, we deal mainly with the geometry of the local singularities.

\section{Characterisations of the singularities of $P_{(l, v)}$}

Given $(l, v) \in \mathcal{C}$ and a point $p \in M$, we denote by $v^{*}$ the tangent at $p$ to the horocycle through $p$ and $q(p,(l, v))$. Then

$$
v^{*}=c^{\prime}\left(s_{0}\right)=v-\frac{\langle p, v\rangle}{\langle p, l\rangle} l
$$

where $c(s)$ and $s_{0}$ are as in the proof of Theorem 3.1. Let $S_{p}^{2}=T_{p} H_{+}^{3}(-1) \cap S_{1}^{3}$, $\mathcal{C}_{p}=\left\{(l, w) \in S_{+}^{2} \times S_{p}^{2} \mid\langle l, w\rangle=0\right\}$ and

$$
\begin{aligned}
& F_{p}: \quad \mathcal{C} \rightarrow \mathcal{C}_{p} \\
& (l, v) \mapsto\left(l, v^{*}\right)
\end{aligned}
$$

Proposition 4.1 The map $F_{p}$ is a diffeomorphism.

Proof. The map $F_{p}$ is clearly of class $C^{\infty}$. It is injective as the vector $v$ is spacelike and $l$ is lightlike. To show that it is surjective, let $(l, w) \in \mathcal{C}_{p}$ and consider the horocycle $c(s)=p+s w-s^{2} /(2\langle p, l\rangle) l$ through $p$. We are seeking a vector $v=$ $w-\left(s_{1} /\langle p, l\rangle\right) l$ tangent to the horocycle at $c\left(s_{1}\right)$ and orthogonal to a plane that passes through $p_{0}=(1,0,0,0)$. So we require $\left\langle p_{0}, v\right\rangle=0$. This gives $s_{1}=\left\langle p_{0}, w\right\rangle\langle p, l\rangle /\left\langle p_{0}, l\right\rangle$ and $v=w-\left\langle p_{0}, w\right\rangle /\left\langle p_{0}, l\right\rangle l$. The inverse map $F_{p}^{-1}(l, w)=\left(l, w-\left\langle p_{0}, w\right\rangle /\left\langle p_{0}, l\right\rangle l\right)$ is clearly of class $C^{\infty}$. 
Let $p$ be a point on an embedded surface $M$ in $H_{+}^{3}(-1)$ and $w \in T_{p} M$. Then as a consequence of Proposition 4.1, if $(l, w) \in \mathcal{C}_{p}$, there is a unique $v \in S_{0}^{2}$ such that $w=v^{*}$.

We have the following geometric characterisations of the singularities of $P_{(l, v)}$, where "worse" means more degenerate, alternatively, has a higher $\mathcal{A}_{e}$-codimension.

Theorem 4.2 Let $M$ be an embedded surface in $H_{+}^{3}(-1)$ and $(l, v) \in \mathcal{C}$.

(1) The projection $P_{(l, v)}$ is singular at $p \in M$ if and only if $v^{*} \in T_{p} M$.

(2) For $v \in S_{0}^{2}$ fixed and $p \in M$, there is a circle of directions $l \in S_{+}^{2} \subset L C^{*}$ such that $v^{*} \in T_{p} M$. This circle contains the directions $\widetilde{\mathbb{L}}^{ \pm}(p)=\widetilde{p \pm e(p)}$.

(3) The projection $P_{(l, v)}$ has a singularity of type cusp or worse at $p \in M$ if and only if $v^{*} \in T_{p} M$ and $\kappa\left(v^{*}\right)=-\lambda(p, l)$, where $\lambda(p, l)=\langle e(p), l\rangle /\langle p, l\rangle$. So, for $l$ fixed, there are at most two directions $v^{*} \in T_{p} M$ for which the singularity of $P_{(l, v)}$ is of type cusp or worse.

(4) The projection $P_{(l, v)}$ has a singularity of type lips/beaks or worse at $p \in M$ if and only if $v^{*}$ is a principal direction and $-\lambda(p, l)$ is its associated de Sitter principal curvature. There are two directions $l$ that satisfy $\kappa_{1}=-\lambda(p, l)\left(\right.$ or $\left.\kappa_{2}=-\lambda(p, l)\right)$.

(5) The curves $\kappa_{i}=$ constant, $i=1,2$, are the loci of points where the two directions in (3) coincide. They foliate the region $-1 \leq \kappa_{i} \leq 1, i=1,2$. (Recall that the curves $\kappa_{i}= \pm 1, i=1,2$, are part of the horo-parabolic set associated to $K_{h}^{ \pm}$.)

(6) The equality $\kappa\left(v^{*}\right)=-\lambda(p, l)= \pm 1$ holds if and only if $l=\widetilde{\mathbb{L}}^{ \pm}(p)$ and $v^{*}$ is a horo-asymptotic direction. So the projection $P_{(\widetilde{\mathbb{L}} \pm(p), v)}$ has a singularity of type cusp at $p$ or worse if and only if $v^{*}$ is a horo-asymptotic direction. The singularity is of type lips/beaks or worse if and only if $p$ is a horo-parabolic point and $v^{*}$ is the unique horo-asymptotic direction at $p$ (which is also a principal direction).

(7) Let $p$ be an umbilic point, $l \in S_{+}^{2}$ with $\left|\kappa_{1}\right|<1$. Then the projection $P_{(l, v)}$ has a singularity of type lips/beaks or worse for any $v \in S_{0}^{2}$ with $v^{*} \in T_{p} M$ and $l \in S_{+}^{2}$ with $-\lambda(p, l)=\kappa_{1}$. For all directions $v^{*} \in T_{p} M$, except a finite number of them, the singularity is genuinely of type lips/beaks. There are generically 0,2,4,6 directions in $T_{p} M$ where the singularity becomes of type $11_{5}$ and $0,2,4,6,8$ where it becomes of type $4_{3}$.

Proof. The statements in the theorem are of local nature, so we shall take the surface $M$ in hyperbolic Monge form (H-Monge form, see [19]) at the point in consideration. In fact, by hyperbolic motions, we can suppose that the point of interest is $p_{0}=(1,0,0,0)$ and the surface given locally by

$$
\boldsymbol{x}(x, y)=\left(\sqrt{f^{2}(x, y)+x^{2}+y^{2}+1}, f(x, y), x, y\right),
$$

with $(x, y)$ in some neighbourhood of the origin. Here $f$ is a smooth function with $f(0,0)=0$ and $f_{x}(0,0)=f_{y}(0,0)=0$. So a unit normal to $M$ at $p_{0}$ is given by 
$e(0,0)=(0,1,0,0)$. We shall write the Taylor expansion of $f$, at the origin, in the form

$$
f(x, y)=a_{20} x^{2}+a_{21} x y+a_{22} y^{2}+\sum_{i=0}^{3} a_{3 i} x^{3-i} y^{i}+\sum_{i=0}^{4} a_{4 i} x^{4-i} y^{i}+\text { h.o.t. }
$$

Let $v=\left(0, v_{1}, v_{2}, v_{3}\right) \in S_{+}^{2}$. In order to make the contribution of $\left\langle p_{0}, l\right\rangle$ apparent, we take $l$ on a sphere in $L C^{*}$ with $l_{0}=$ constant. Then $l_{0}=\left\langle p_{0}, l\right\rangle$. (If $l \in S_{+}^{2}$, then $\left\langle p_{0}, l\right\rangle=1$.) We assume that $\langle l, v\rangle=0$. We have $v^{*}=v$ at $p_{0}$ and

$$
\begin{aligned}
& \partial P_{(l, v)} / \partial x(0,0)=\left(0,-v_{1} v_{2}, 1-v_{2}^{2},-v_{2} v_{3}\right) \\
& \partial P_{(l, v)} / \partial y(0,0)=\left(0,-v_{1} v_{3},-v_{2} v_{3}, 1-v_{3}^{2}\right) .
\end{aligned}
$$

These two vectors are linearly dependent if and only if $v_{1}=0$, if and only if $v^{*} \in T_{p_{0}} M$, which proves (1).

(2) We suppose from now on that $v_{1}=0$. Then $v_{2}^{2}+v_{3}^{2}=1$ and $l=\left(l_{0}, l_{1},-t v_{3}, t v_{2}\right)$ with $l_{1}^{2}+t^{2}=l_{0}^{2}$. We still denote $\widetilde{\mathbb{L}}^{ \pm}(p)$ the projection of $p+e(p)$ to the sphere $l_{0}=$ constant. We have $\widetilde{\mathbb{L}}^{ \pm}\left(p_{0}\right)=\left(l_{0}, \pm l_{1}, 0,0\right)$, so $\left\langle\widetilde{\mathbb{L}}^{ \pm}\left(p_{0}\right), v\right\rangle=0$ for any $v=v^{*} \in T_{p_{0}} M$.

(3), (4) and (5) We write $P_{(l, v)}[i]$ for the $i$ th coordinate function of $P_{(l, v)}$. The tangent plane $\Pi$ of $H P(v, 0) \cap H_{+}^{3}(-1)$ at $p_{0}$ is generated by the two vectors $(0,1,0,0)$ and $\left(0,0,-v_{3}, v_{2}\right)$. Let $\pi: H P(v, 0) \cap H_{+}^{3}(-1) \rightarrow \Pi$ denotes the linear projection along the vector $v$. Consider the composite map

$$
\pi \circ P_{(l, v)}=\left(0, P_{(l, v)}[2], v_{3}\left(v_{3} P_{(l, v)}[3]-v_{2} P_{(l, v)}[4]\right),-v_{2}\left(v_{3} P_{(l, v)}[3]-v_{2} P_{(l, v)}[4]\right)\right),
$$

which is $\mathcal{A}$-equivalent to the map-germ $\mathbb{R}^{2}, 0 \rightarrow \mathbb{R}^{2}, 0$ given by

$$
\widetilde{P}_{(l, v)}=\left(P_{(l, v)}[2], v_{3} P_{(l, v)}[3]-v_{2} P_{(l, v)}[4]\right) .
$$

As the singularities of $P_{(l, v)}$ restricted to $M$ and those of $\widetilde{P}_{(l, v)}$ are $\mathcal{A}$-equivalent, we work with the map-germ $\widetilde{P}_{(l, v)}$. We reduce the appropriate $k$-jets of $\widetilde{P}_{(l, v)}$ to the form $(x, g(x, y))$ and interpret geometrically the conditions for this germ to be $\mathcal{A}$-equivalent to one in Table 1 . A calculation shows that

$$
j^{2} \widetilde{P}_{(l, v)} \sim_{\mathcal{A}}\left(x,\left(2 l_{0}\left(a_{20} v_{2}^{2}+a_{21} v_{2} v_{3}+a_{22} v_{3}^{2}\right)-l_{1}\right) y^{2}+\left(\left(2 a_{20} v_{2}+v_{3} a_{21}\right) l_{0}-v_{2} l_{1}\right) x y\right) .
$$

The singularity of $P_{(l, v)}$ at $p_{0}$ is of type cusp or worse if and only if the coefficient of $y^{2}$ in the above expression vanishes. This is the case if and only if

$$
2\left(a_{20} v_{2}^{2}+a_{21} v_{2} v_{3}+a_{22} v_{3}^{2}\right)=\frac{l_{1}}{l_{0}}
$$

which can be written as

$$
\kappa\left(v^{*}\right)=-\frac{\left\langle e\left(p_{0}\right), l\right\rangle}{\left\langle p_{0}, l\right\rangle} .
$$


Since $l \in L C^{*}, l_{1}^{2}+l_{2}^{2}+l_{3}^{2}=l_{0}^{2}$, so $\left|l_{1} / l_{0}\right| \leq 1$. (Alternatively, we can choose $p_{0}, e\left(p_{0}\right), v^{*}, v^{* \perp}$ as an orthonormal basis of $\mathbb{R}_{1}^{4}$, where $v^{* \perp}$ is a unit orthogonal vector to $v^{*}$ in $T_{p_{0}} M$. We have $\left\langle l, v^{*}\right\rangle=0$, so $l=\left\langle p_{0}, l\right\rangle p_{0}+\left\langle e\left(p_{0}\right), l\right\rangle e\left(p_{0}\right)+\left\langle v^{* \perp}, l\right\rangle v^{* \perp}$. But $l \in L C^{*}$, so $-\left\langle p_{0}, l\right\rangle^{2}+\left\langle e\left(p_{0}\right), l\right\rangle^{2}+\left\langle v^{* \perp}, l\right\rangle^{2}=0$, which gives $\left|\left\langle e\left(p_{0}\right), l\right\rangle /\left\langle p_{0}, l\right\rangle\right| \leq 1$.)

Now, $\kappa\left(v^{*}\right)=\cos ^{2} \theta \kappa_{1}+\sin ^{2} \theta \kappa_{2}$, with $\theta$ the angle $v^{*}$ makes with the principal direction $p_{1}$ associated to $\kappa_{1}$. Therefore $\kappa_{1} \leq \kappa\left(v^{*}\right) \leq \kappa_{2}$, so for equation (1) to have a solution (in $v$ or $l$ ) we need $m=\min \left(\left|\kappa_{1}\right|,\left|\kappa_{2}\right|\right) \leq 1$. If $m>\left|\lambda\left(p_{0}, l\right)\right|$ where $\lambda\left(p_{0}, l\right)=\left\langle e\left(p_{0}\right), l\right\rangle /\left\langle p_{0}, l\right\rangle=-l_{1} / l_{0}$, then there are no solutions to $\kappa\left(v^{*}\right)=-\lambda\left(p_{0}, l\right)$. If $m<\left|\lambda\left(p_{0}, l\right)\right|$, for any $\lambda$ there are two directions in the tangent space that satisfy the equation $\kappa\left(v^{*}\right)=-\lambda\left(p_{0}, l\right)$. A short calculation shows that the two directions coincide if and only if

$$
\left(\frac{l_{1}}{l_{0}}\right)^{2}-2\left(a_{22}+a_{20}\right) \frac{l_{1}}{l_{0}}+4 a_{20} a_{22}-a_{21}^{2}=0 .
$$

This means that $-\lambda\left(p_{0}, l\right)$ is a de Sitter principal curvature, and the direction $v^{*}$ is the associated principal direction. When this is the case, the coefficient of $x y$ in the expression for the 2 -jet of $\widetilde{P}_{(l, v)}$ also vanishes. So the singularity becomes of type lips/beaks or worse.

(6) In the above setting, if $\left|\lambda\left(p_{0}, l\right)\right|=1$, then $\left\langle v^{* \perp}, l\right\rangle=0$. This means that $l=\left\langle p_{0}, l\right\rangle\left(p_{0} \pm e\left(p_{0}\right)\right)$, therefore $\widetilde{l}=\widetilde{\mathbb{L}}^{ \pm}\left(p_{0}\right)$.

We know that $\bar{\kappa}^{ \pm}\left(v^{*}\right)=-1 \pm \kappa\left(v^{*}\right)$, so $\kappa\left(v^{*}\right)= \pm 1$ if and only if $\bar{\kappa}^{ \pm}\left(v^{*}\right)=0$, if and only if $v^{*}$ is a horo-asymptotic direction.

(7) Follows by analysing the 3 -jet of $\widetilde{P}_{(l, v)}$ at an umbilic point.

Proposition 4.3 The projection $P_{\left(\widetilde{\mathbb{L}}^{ \pm}(p), v\right)}$ has a singularity of type swallowtail $\left(11_{5}\right)$ or worse at $p$ if and only if $v^{*}$ is a horo-asymptotic direction and $p$ is a geodesic inflection on the associated horo-asymptotic direction. The locus of such points form generically a smooth curve on $M$ that we label the horo-flecnodal curve of $M$.

Proof. We choose the setting of the proof of Theorem 4.2, take $p=p_{0}$ and analyse the 3 -jet of $P_{\left(\widetilde{\mathbb{L}}^{ \pm}\left(p_{0}\right), v\right)}$. We write this 3-jet in the form $(x, g(x, y))$. Then $p_{0}$ is a is swallowtail singularity (or worse) of $P_{\left(\widetilde{\mathbb{L}} \pm\left(p_{0}\right), v\right)}$, if and only if $\partial^{2} g / \partial y^{2}(0,0)=\partial^{3} g / \partial y^{2}(0,0)=0$. Calculations show that this is the case if and only if

$$
\left(1 \pm 2 a_{20}\right) v_{2}^{2} \pm 2 a_{21} v_{2} v_{3}+\left(1 \pm 2 a_{22}\right) v_{3}^{2}=0
$$

(i.e., $v^{*}=\left(0,0, v_{2}, v_{3}\right)$ is a horo-asymptotic direction) and

$$
C\left(v_{2}, v_{3}\right)=a_{30} v_{2}^{3}+a_{31} v_{2}^{2} v_{3}+a_{32} v_{2} v_{3}^{2}+a_{33} v_{3}^{2}=0
$$

where $C$ is the cubic part of $f$. To simplify the calculations, we rotate the axis in the tangent plane and suppose that $(0,0,1,0)$ is a horo-asymptotic direction, so 
$1 \pm 2 a_{20}=0$. Then the singularity is of type swallowtail or worse if and only if $a_{30}=0$.

The equation of the horo-asymptotic curves in the parameter space is given by $\bar{l} d x^{2}+2 \bar{m} d x d y+\bar{n} d y^{2}=0$, where $\bar{l}, \bar{m}, \bar{n}$ are the coefficients of the hyperbolic second fundamental form. Then the horo-asymptotic curve tangent to $v^{*}=(0,0,1,0)$ is parametrised by

$$
\gamma(t)=\left(1+\frac{1}{2} t^{2}, \mp \frac{1}{2} t^{2}, t,-\frac{3}{2} \frac{a_{30}}{a_{21}} t^{2}\right)+\text { h.o.t. }
$$

The geodesic curvature of this asymptotic curve at $p_{0}$ is $3 a_{30} /\left(2 a_{21}\right)$. This vanishes when $a_{30}=0$, equivalently when the singularity of the projection is of type swallowtail or worse.

To show that the horo-flecnodal curve is generically a smooth curve, one can follow the method in [5] (see also [8]) and consider the Monge-Taylor map $\phi: U \rightarrow J^{3}(2)$ which associates to $(x, y) \in U$ the 3 -jet of $f$ at $(x, y)$, where $f$ is the function in the parametrisation of the surface $\boldsymbol{x}(U)$ in H-Monge form. The swallowtail singularities form a smooth variety in $J^{3}(2)$ and for generic surfaces $\phi(U)$ intersects this variety transversally. The horo-flecnodal curve is the pre-image of this intersection, so is generically a smooth curve.

We call the image of the critical set of $P_{(l, v)}$ the contour (or profile) of $M$. We shall suppose here that it is a smooth curve and restrict to the case where $l=\widetilde{\mathbb{L}}^{ \pm}\left(p_{0}\right)$ at some point $p_{0} \in M$. We call the intersection of $M$ with the 3-dimensional space generated by the vectors $p_{0}, v^{*}$ and $e\left(p_{0}\right)$ the normal section of $M$ at $p_{0}$ along $v^{*}$. Koenderink showed in [30] that for embedded surfaces in $\mathbb{R}^{3}$, the Gaussian curvature of the surface at a given point is the product of the curvature of the contour with the curvature of the normal section in the direction of projection. We have the following result for the projections $P_{\left(\widetilde{\mathbb{L}}^{ \pm}\left(p_{0}\right), v\right)}$, where the curvature of a curve in $H_{+}^{3}(-1)$ is as given in $\S 3$.

Theorem 4.4 (Koenderink type theorem) Let $\kappa_{c}$ be the curvature of the contour and $\kappa_{n}$ the curvature of the normal section. If the point on the surface is also on the plane of projection (alternatively, if $v \in T_{p} M$ ), then

$$
K_{h}^{ \pm}=\left(\kappa_{c}+1\right)\left(\kappa_{n} \mp 1\right) \text {. }
$$

Otherwise, the left hand side of the above equality depends on $\left\langle v^{*}, p\right\rangle$.

Proof. We take, without loss of generality, the surface in the H-Monge form as in the proof of Theorem 4.2, $v=v^{*}=(0,0,0,1)$ and $l=(1, \pm 1,0,0)$ at $p_{0}=(1,0,0,0)$. We assume that the singularity of the projection is a fold at $p_{0}$, so $2 a_{22} \pm 1 \neq 0$. Then the 2 -jet of the profile is given by

$$
\left(1+\frac{1}{2} t^{2}, \frac{\left(4 a_{20} a_{22}-2 a_{20}-a_{21}^{2}\right)}{2\left(2 a_{22}-1\right)} t^{2}, t, 0\right) .
$$


Following the formula in $\S 3$, its curvature at $p_{0}$ is given by

$$
\kappa_{c}=\frac{4 a_{20} a_{22}-a_{21}^{2} \mp 2 a_{20}}{2 a_{22} \mp 1} .
$$

The normal section of the surface along $v$ is given by $\left(\sqrt{f(0, y)^{2}+y^{2}+1}, f(0, y), 0, y\right)$ and its curvature at $p_{0}$ is given by $\kappa_{n}=2 a_{22}$. Given the fact that the hyperbolic curvature of the surface at $p_{0}$ is $K_{h}^{ \pm}=4 a_{20} a_{22}-a_{21}^{2} \mp 2 a_{20} \mp 2 a_{22}+1$, it follows that

$$
\kappa_{c}=\frac{K_{h}^{ \pm}}{\kappa_{n} \mp 1}-1
$$

equivalently,

$$
K_{h}^{ \pm}=\left(\kappa_{c}+1\right)\left(\kappa_{n} \mp 1\right) .
$$

We now show that the above formula does not hold if $p_{0}$ does not belong to the plane of projection. Let $\lambda \in \mathbb{R}$ and consider the map

$$
\begin{array}{ccc}
h c: \quad H P(v, 0) \cap H_{+}^{3}(-1) & \rightarrow H P(v, \lambda) \cap H_{+}^{3}(-1) \\
q & \mapsto \quad q+\lambda v-\frac{\lambda^{2}}{2\langle q, l\rangle} l
\end{array}
$$

that takes a point on the plane determined by $v \in S_{0}^{2}$ to a point on another parallel plane along the horocycles $(l, v) \in \mathcal{C}$. It is not difficult to show that the curvature of a curve $\gamma(t) \subset H P(v, 0) \cap H_{+}^{3}(-1)$ is distinct from that of $h c(\gamma(t)) \subset H P(v, \lambda) \cap H_{+}^{3}(-1)$ when $\lambda \neq 0$. So if we project to $H P(v, \lambda) \cap H_{+}^{3}(-1)$ instead, the left hand side of the above equality remains the same while the value of $\kappa_{c}$ changes as $\lambda$ varies. So the equality does not hold in this case.

\section{Duality}

We prove in this section duality result similar to those in [34] for central projections of surfaces in $\mathbb{R} P^{3}$ and to those in [9] for orthogonal projections of surfaces in $\mathbb{R}^{3}$.

Let $M$ be an embedded surface in $H_{+}^{3}(-1)$. We shall use the duality concepts in $[13,14,25]$, see $\S 6$ for details. In [24] is introduced the notion of a horocyclic surface which is defined to be a one-parmeter family of horocylces in $H_{+}^{3}(-1)$. We denote by $A_{2}^{h-p a r}$ the horocyclic surface in $H_{+}^{3}(-1)$ swept out by the horocycles in $H_{+}^{3}(-1)$ passing through a horo-parabolic point of $M$ and with tangent direction there the unique horoasymptotic direction. We also denote by $\left(A_{2}^{h-p a r}\right)^{(1, *)}$ the $\Delta_{1}$-dual of $A_{2}^{h-p a r}$.

A bi-tangent horosphere of $M$ is a horosphere $H S^{3}(l, c)$ tangent to $M$ at two distinct points. If there exist points $p_{1}, p_{2} \in M$ such that $\mathbb{L}^{ \pm}\left(p_{1}\right)=\mathbb{L}^{ \pm}\left(p_{2}\right)$ and $\left\langle p_{1}, \mathbb{L}^{ \pm}\left(p_{1}\right)\right\rangle=$ $\left\langle p_{2}, \mathbb{L}^{ \pm}\left(p_{2}\right)\right\rangle$, then we have the bi-tangent horosphere $H S^{3}\left(\mathbb{L}^{ \pm}\left(p_{1}\right),\left\langle p_{1}, \mathbb{L}^{ \pm}\left(p_{1}\right)\right\rangle\right)$ at $p_{1}$ and $p_{2}$. In this case, there exists a unique horocycle in $H S^{3}\left(\mathbb{L}^{ \pm}\left(p_{1}\right),\left\langle p_{1}, \mathbb{L}^{ \pm}\left(p_{1}\right)\right\rangle\right)$ 
passing through $p_{1}$ and $p_{2}$. We call such horocycle a bi-tangent horocycle to $M$ relative to $\mathbb{L}^{ \pm}\left(p_{1}\right)=\mathbb{L}^{ \pm}\left(p_{2}\right)$. We denote by $A_{1}^{h} \| A_{1}^{h}$ the horocyclic surface swept out by the bitangent horocycles to $M$ relative to the lightcone normal at points on the bi-tangent locus of horospheres to $M$.

Theorem 5.1 Let $M$ be an embedded surface in $H_{+}^{3}(-1)$ and $M^{(2, *)}$ its $\Delta_{2}$-dual. Then,

(1) The $\Delta_{2}$-dual of the surface $A_{2}^{h-p a r}$ is the cuspidaledge of $M^{(2, *)}$.

(2) The $\Delta_{2}$-dual of the surface $A_{1}^{h} \| A_{1}^{h}$ is the self-intersection curve of $M^{(2, *)}$.

(3) The $\Delta_{3}$-dual of the surface $\left(A_{2}^{h-p a r}\right)^{(1, *)}$ is the cuspidaledge of $-M^{(2, *)}$, where $-M^{(2, *)}$ denotes the antipodal surface of $M^{(2, *)}$.

Proof. We consider a local parametrisation $\boldsymbol{x}: U \rightarrow H_{+}^{3}(-1)$ of $M$ (i.e., $M=\boldsymbol{x}(U)$ ). In this case $M^{(2, *)}=\mathbb{L}^{ \pm}(U)$.

(1) We suppose that the horo-parabolic set $K_{h}^{ \pm}=0$ is a regular curve. This property holds for generic embeddings of surface $M$ in $H_{+}^{3}(-1)$. Let $p(t), t \in I$, be a parametrisation of the horo-parabolic set of $M$ and $u(t)$ the unique unit horoasymptotic direction at $p(t)$. There exists a curve $\gamma: I \rightarrow U$ such that $\boldsymbol{x}(\gamma(t))=p(t)$ and $u(t) \in T_{\gamma(t)} M$. Then we have $e(t)=\mathbb{E}(\gamma(t))$, so that $\mathbb{L}^{ \pm}(\gamma(t))=p(t) \pm e(t)$. Since $p(t)=\boldsymbol{x}(\gamma(t))$ is a parametrisation of $K_{h}^{-1}(0)$ which is the singular locus of $\mathbb{L}^{ \pm}$, $\mathbb{L}^{ \pm}(\gamma(t))=p(t) \pm e(t)$ is the cuspidaledge of $M^{(2, *)}=\mathbb{L}^{ \pm}(U)$.

The horocyclic surface $A_{2}^{h-p a r}$ is parametrised by

$$
y(s, t)=p(t)+s u(t)+\frac{s^{2}}{2}(p(t) \pm e(t)) .
$$

We have

$$
\frac{\partial y}{\partial s}(s, t)=u(t)+s(p(t) \pm e(t)) \quad \text { and } \quad \frac{\partial y}{\partial t}(s, t)=p^{\prime}(t)+s u^{\prime}(t)+\frac{s^{2}}{2}\left(p^{\prime}(t) \pm e^{\prime}(t)\right) .
$$

Since $u(t) \in T_{\gamma(t)} M$ and $\mathbb{L}^{ \pm}(\gamma(t))=p(t) \pm e(t)$ is a lightlike normal to $M$, we have

$$
\left\langle\frac{\partial y}{\partial s}(s, t), \mathbb{L}(\gamma(t))\right\rangle=0 .
$$

We remark that $p^{\prime}(t) \in T_{\gamma(t)} M$ and $\left\langle p^{\prime}(t) \pm e^{\prime}(t), p(t) \pm e(t)\right\rangle=0$. Therefore, we have

$$
\left\langle\frac{\partial y}{\partial t}(s, t), p(t) \pm e(t)\right\rangle=s\left\langle u^{\prime}(t), p(t) \pm e(t)\right\rangle .
$$

Taking the derivative of the relation $\langle p(t) \pm e(t), u(t)\rangle=0$, we have

$$
\left\langle d \mathbb{L}^{ \pm}(\gamma(t)) p^{\prime}(t), u(t)\right\rangle=\left\langle\left(\mathbb{L}^{ \pm}\right)^{\prime}(\gamma(t)), u(t)\right\rangle=-\left\langle p(t) \pm e(t), u^{\prime}(t)\right\rangle .
$$


The fact that $p(t)$ is the parametrisation of horo-parabolic set means that the image of $d \mathbb{L}^{ \pm}(\gamma(t))$ is one dimensional, so that there exists $\lambda \in \mathbb{R}$ such that

$$
d \mathbb{L}^{ \pm}(\gamma(t)) p^{\prime}(t)=\lambda d \mathbb{L}^{ \pm}(\gamma(t)) u(t) .
$$

Since $u(t)$ is the unique asymptotic direction, we have

$$
\left\langle d \mathbb{L}^{ \pm}(\gamma(t)) p^{\prime}(t), u(t)\right\rangle=\lambda\left\langle d \mathbb{L}^{ \pm}(\gamma(t)) u(t), u(t)\right\rangle=0 .
$$

It follows that

$$
\left\langle\frac{\partial y}{\partial t}(s, t), \mathbb{L}^{ \pm}(\gamma(t))\right\rangle=0 .
$$

So the lightlike normal to the horocyclic surface $A_{2}^{h-p a r}$ is constant along each horocycle. (This means that the image of the lightcone normal to $A_{2}^{h-p a r}$ is a curve in $L C^{*}$, which implies that $A_{2}^{h-p a r}$ is a horo-flat horocyclic surface in $H_{+}^{3}(-1)$; see [24].) Moreover, we have $\left\langle y(s, t), \mathbb{L}^{ \pm}(\gamma(t))\right\rangle=-1$. Therefore $y(s, t)$ and $\mathbb{L}^{ \pm}(\gamma(t))$ are $\Delta_{2}$-dual.

(2) Suppose that there exists a bi-tangent horocycle to $M$ at two points $p_{1}$ and $p_{2}$ on $M$. The surface $A_{1}^{h} \| A_{1}^{h}$ is then a horocyclic surface generated by horocycles along a curve $C_{1}$ on $M$ through $p_{1}$ (or a curve $C_{2}$ through $p_{2}$ ). The lightlike normals $\mathbb{L}^{ \pm}$of $M$ along $C_{1}$ and $C_{2}$ coincide. Let $q(t)$ be a local parametrisation of the curve $C_{1}$ and $v(t)$ be the unit tangent direction to the horocycle in $A_{1}^{h} \| A_{1}^{h}$ through $q(t)$. We also denote by $e(t)$ the de Sitter normal to $M$ along $C_{1}$, so that the lightcone normal to $M$ along $C_{1}$ is given by $\mathbb{L}^{ \pm}(t)=q(t) \pm e(t)$. Then a local parametrisation of $A_{1}^{h} \| A_{1}^{h}$ is given by

$$
z(s, t)=q(t)+s v(t)+\frac{s^{2}}{2}(q(t) \pm e(t)) .
$$

It follows that

$$
\frac{\partial z}{\partial s}(s, t)=v(t)+s(q(t) \pm e(t)) \quad \text { and } \quad \frac{\partial z}{\partial t}(s, t)=q^{\prime}(t)+s v^{\prime}(t)+\frac{s^{2}}{2}\left(q^{\prime}(t) \pm e^{\prime}(t)\right) .
$$

By the same arguments in case (1), we have

$$
\left\langle\frac{\partial z}{\partial s}(s, t), q(t) \pm e(t)\right\rangle=0 \quad \text { and } \quad\left\langle\frac{\partial z}{\partial t}(s, t), q(t) \pm e(t)\right\rangle=s\left\langle v^{\prime}(t), q(t) \pm e(t)\right\rangle .
$$

Since $C_{1}$ and $C_{2}$ are disjoint, $C_{2}$ can be parametrised by $q_{2}(t)=z(s(t), t)$ for some non-zero smooth function $s(t)$. On $C_{2}$, we have

$$
0=\left\langle\frac{\partial z}{\partial t}(s(t), t), q(t) \pm e(t)\right\rangle=s(t)\left\langle v^{\prime}(t), q(t) \pm e(t)\right\rangle .
$$

Since $s(t) \neq 0$, we have $\left\langle v^{\prime}(t), q(t) \pm e(t)\right\rangle=0$, so that

$$
\left\langle\frac{\partial z}{\partial t}(s, t), q(t) \pm e(t)\right\rangle=0 .
$$


So the lightlike normal to the horocyclic surface $A_{1}^{h} \| A_{1}^{h}$ is constant along each horocycle. (This means that the image of the lightcone normal to $A_{1}^{h} \| A_{1}^{h}$ is a curve in $L C^{*}$, which implies that $A_{1}^{h} \| A_{1}^{h}$ is a horo-flat horocyclic surface in $H_{+}^{3}(-1)$; see [24].) Moreover, we have $\left\langle z(s, t), \mathbb{L}^{ \pm}(t)\right\rangle=-1$. This means that $z(s, t)$ and $\mathbb{L}^{ \pm}(t)$ are $\Delta_{2}$-dual.

The maps $q(t)=z(t, 0)$ and $z(t, s(t))$ are parametrisations of $C_{1}$ and $C_{2}$ respectively. Therefore, $\mathbb{L}^{ \pm}(t)$ is the common lightcone normal to $M$ at $q(t)$ and $z(t, s(t))$, which is the self intersection curve of $M^{(2, *)}$.

(3) We use here the notation in case (1) and define the contact diffeomorphism $\Psi_{31}: \Delta_{3} \rightarrow \Delta_{1}$ by $\Psi_{31}(v, w)=(v-w,-w)$. We also define the mapping

$$
w(s, t)= \pm e(t)-s u(t)-\frac{s^{2}}{2}(p(t) \pm e(t)) .
$$

One can easily show that $w(s, t) \in S_{1}^{3}$ and $\langle p(t) \pm e(t), w(s, t)\rangle=1$. By almost the same calculations as those in case $(1)$, we can show that

$$
\left\langle\frac{\partial w}{\partial s}(s, t), q(t) \pm e(t)\right\rangle=0 \quad \text { and } \quad\left\langle\frac{\partial w}{\partial t}(s, t), q(t) \pm e(t)\right\rangle=0 .
$$

This means that $\mathbb{L}^{ \pm}(\gamma(t))=p(t) \pm e(t)$ and $w(s, t)$ are $\Delta_{3}$-dual. We also have

$$
\Psi_{31}\left(\mathbb{L}^{ \pm}(\gamma(t)), w(s, t)\right)=(y(s, t),-w(s, t)) .
$$

Therefore $y(s, t)$ is the $\Delta_{1}$-dual of $-w(s, t)$. Since $y(s, t)$ gives a local parametrisation of $A_{2}^{h-p a r},-w(s, t)$ is a local parametrisation of $\left(A_{2}^{h-p a r}\right)^{(1, *)}$. By definition, $\Delta_{3}$ is invariant under the antipodal action on $\mathbb{R}_{1}^{4}$, so $\left(A_{2}^{h-p a r}\right)^{(1, *)}$ is the $\Delta_{3}$-dual of $-\mathbb{L}^{ \pm}(\gamma(t))$ which is the cuspidaledge of $-M^{(2, *)}$.

There are Euclidean analogues in [9] of the results in [34] (see also [3, 4, 7] for related results). Given an embedded surface $M$ in the Euclidean space $\mathbb{R}^{3}$ the family of height functions on $M$ is given by

$$
\begin{aligned}
H_{E}: M \times S^{2} & \rightarrow \mathbb{R} \times S^{2} \\
(q, v) & \mapsto q \cdot v
\end{aligned}
$$

and the family of orthogonal projections is given by

$$
\begin{array}{rlc}
P_{E}: M \times S^{2} & \rightarrow & T S^{2} \\
(q, v) & \mapsto & (q, q-(q \cdot v) v)
\end{array}
$$

where $S^{2}$ denotes the unit sphere and "." the scalar product in $\mathbb{R}^{3}$. The local bifurcation set $B i f\left(H_{E}\right)$ of $H_{E}$ (resp. Bif $\left(P_{E}\right)$ of $P_{E}$ ) is the set of $u \in S^{2}$ for which there exist $p \in M$ such that $H_{E}(-, u)$ (resp. $P_{E}(-, u)$ ) has a non-stable singularity at $p$. The $A_{2}$-stratum of $\operatorname{Bif}\left(H_{E}\right)$ is the set of unit normals at the parabolic points of 
$M$. The lips/beaks stratum of $\operatorname{Bif}\left(P_{E}\right)$ is the set of unit asymptotic directions at the parabolic points of $M$. It is shown in [9] that the $A_{2}$-stratum of $B i f\left(H_{E}\right)$ is dual to the lips/beaks stratum of Bif $\left(P_{E}\right)$. The duality in [9] refers to the double Legendrian fibration $S^{2} \stackrel{\pi_{1}}{\longleftarrow} \Delta \stackrel{\pi_{2}}{\longrightarrow} S^{2}$, where $\Delta=\left\{(u, v) \in S^{2} \times S^{2} \mid u . v=0\right\}$. The contact structure on $\Delta$ is given by the 1 -form $\theta=\left.v \cdot d u\right|_{\Delta}$.

We seek an analogous duality result for the family of projections along horocycles. The family of lightcone height functions on $M \subset H_{+}^{3}(-1)$ is introduced in [19] and is given by

$$
\begin{aligned}
H: M \times S_{+}^{2} & \rightarrow \mathbb{R} \times S_{+}^{2} \\
(p, l) & \mapsto\langle p, l\rangle
\end{aligned}
$$

The $A_{2}$-stratum of $\operatorname{Bif}(H)$ is the set of lightcone vectors $\widetilde{\mathbb{L}}^{ \pm}(p)$, with $p$ a horo-parabolic point of $M$. We know from Theorem 4.2 that the projection $P_{\left.\widetilde{\mathbb{L}}^{ \pm}(p), v\right)}$ has a lips/beaks singularity when $p$ a horo-parabolic point and $v^{*}$ is a horo-asymptotic direction at $p$.

The set $\mathcal{C}=\left\{(l, v) \in S_{+}^{2} \times S_{0}^{2} \mid\langle l, v\rangle=0\right\}$ can be given a contact structure associated to the 1 -form $\theta=\left.\langle v, d l\rangle\right|_{\mathcal{C}}$. The smooth fibre bundles $\pi_{1}: \mathcal{C} \rightarrow S_{+}^{2}$ and $\pi_{2}: \mathcal{C} \rightarrow S_{0}^{2}$ are Legendrian fibrations. Therefore, given a Legendrian curve $\boldsymbol{i}: L \rightarrow \mathcal{C}, \pi_{1}(\boldsymbol{i}(L))$ is dual to $\pi_{2}(\boldsymbol{i}(L))$ and vice-versa. We call this duality $\mathcal{C}$-duality. (See $\S 6$ for more details on Legendrian duality.)

Suppose that the horo-parabolic set of $M \in H_{+}^{3}(-1)$ is smooth (which is generically the case) and is parametrised locally by $p(t), t \in I$. Let $u(t)$ be the unique horoasymptotic direction at $p(t)$ and $\left(\widetilde{\mathbb{L}}^{ \pm}(p(t)), v(t)\right)=F_{p(t)}^{-1}\left(\widetilde{\mathbb{L}}^{ \pm}(p(t)), u(t)\right)$, with $F_{p}$ the map in Proposition 4.1. We have the following consequence of the $\mathcal{C}$-duality.

Proposition 5.2 The curve $L=\left\{\left(\widetilde{\mathbb{L}}^{ \pm}(p(t)), v(t)\right), t \in I\right\}$ is a Legendrian curve in $\mathcal{C}$. Therefore $\pi_{1}(L)$ and $\pi_{2}(L)$ are $\mathcal{C}$-dual curves. That is, the $A_{2}$-stratum of the bifurcation set of the family of lightcone height functions is $\mathcal{C}$-dual to the set of unit horo-asymptotic directions at horo-parabolic points transported along horocycles to $S_{0}^{2}$.

The set of unit horo-asymptotic directions at horo-parabolic points transported along horocycles to $S_{0}^{2}$ is not of course the lips/beaks stratum of the bifurcation set of the family of projections along horocycles. The lips/beaks stratum of this family is generically a 2-dimensional surface in $\mathcal{C}$. However, the curve $L=\left\{\left(\widetilde{L}^{ \pm}(p(t)), v(t)\right), t \in\right.$ $I\}$ is special on this stratum. To show this, let

$$
S\left(4_{2}\right)=\left\{(p,(l, v)) \in M \times \mathcal{C} \mid P_{(l, v)} \text { has a singularity at } p \text { of type lips/beaks }\right\},
$$

The surface $S\left(4_{2}\right)$ is generically smooth. Consider the projections

$$
\pi_{1}: S\left(4_{2}\right) \subset M \times \mathcal{C} \rightarrow M \text { and } \pi_{2}: S\left(4_{2}\right) \subset M \times \mathcal{C} \rightarrow \mathcal{C} .
$$

Proposition 5.3 Suppose that $p$ is not an umbilic point. Then the projection $\pi_{1}$ is singular if and only if $p$ is a horo-parabolic point, $l=\widetilde{L}^{ \pm}(p)$ and $v^{*}$ is the unique horo-asymptotic direction at $p$. Therefore $L=\left\{\left(\widetilde{\mathbb{L}}^{ \pm}(p(t)), v(t)\right), t \in I\right\}=\pi_{2}\left(\Sigma\left(\pi_{1}\right)\right)$. 
Proof. The proof follows by direct calculations using the H-Monge form setting.

\section{Appendix}

We require some properties of contact manifolds and Legendrian submanifolds for the duality results in this paper (for more details see for example [2]). Let $N$ be a $(2 n+1)$ dimensional smooth manifold and $K$ be a field of tangent hyperplanes on $N$. Such a field is locally defined by a 1-form $\alpha$. The tangent hyperplane field $K$ is said to be non-degenerate if $\alpha \wedge(d \alpha)^{n} \neq 0$ at any point on $N$. The pair $(N, K)$ is a contact manifold if $K$ is a non-degenerate hyperplane field. In this case $K$ is called a contact structure and $\alpha$ a contact form.

A submanifold $\boldsymbol{i}: L \subset N$ of a contact manifold $(N, K)$ is said to be Legendrian if $\operatorname{dim} L=n$ and $d \boldsymbol{i}_{x}\left(T_{x} L\right) \subset K_{\boldsymbol{i}_{(x)}}$ at any $x \in L$. A smooth fibre bundle $\pi: E \rightarrow M$ is called a Legendrian fibration if its total space $E$ is furnished with a contact structure and the fibres of $\pi$ are Legendrian submanifolds. Let $\pi: E \rightarrow M$ be a Legendrian fibration. For a Legendrian submanifold $\boldsymbol{i}: L \subset E, \pi \circ \boldsymbol{i}: L \rightarrow M$ is called a Legendrian map. The image of the Legendrian map $\pi \circ \boldsymbol{i}$ is called a wavefront set of $\boldsymbol{i}$ and is denoted by $W(\boldsymbol{i})$.

The duality concepts we use in this paper are those introduced in [13, 14, 25], where five Legendrian double fibrations are considered on the subsets $\Delta_{i}, i=1, \ldots, 5$ below, of the product of two of the pseudo spheres $H^{n}(-1), S_{1}^{n}$ and $L C^{*}$. The geometric ideas behind the choice of the subsets $\Delta_{i}$ and the Legendrian double fibrations are as follows (for more details see $[13,14,25]$ ).

To any hypersurface $\boldsymbol{x}: U \rightarrow H^{n}(-1)$ is associated the de Sitter Gauss map $\mathbb{E}: U \rightarrow S_{1}^{n}$. It is easy to show that the pair $(\boldsymbol{x}, \mathbb{E}): U \rightarrow H^{n}(-1) \times S_{1}^{n}$ is a Legendrian embedding into the set $\Delta_{1}=\left\{(v, w) \in H^{n}(-1) \times S_{1}^{n} \mid\langle v, w\rangle=0\right\}$. (The contact structure on $\Delta_{1}$ is given below.) This means that $M=\boldsymbol{x}(U)$ and $M^{(1, *)}=\mathbb{E}(U)$ are dual. We call this duality the $\Delta_{1}$-duality. This is a direct analogue of the spherical duality in the Euclidean space.

Consider now the lightcone Gauss map $\mathbb{L}^{ \pm}: U \rightarrow H^{n}(-1) \times L C^{*}$ which satisfies $\left\langle\boldsymbol{x}(u), \mathbb{L}^{ \pm}(u)\right\rangle=-1$. The pair $\left(\boldsymbol{x}, \mathbb{L}^{ \pm}\right): U \rightarrow H^{n}(-1) \times L C^{*}$ determines a Legendrian embedding into the set $\Delta_{2}=\left\{(v, w) \in H^{n}(-1) \times L C^{*} \mid\langle v, w\rangle=-1\right\}$, so $M=\boldsymbol{x}(U)$ and $M^{(2, *)}=\mathbb{L}^{ \pm}(U)$ are dual. We call this duality the $\Delta_{2}$-duality.

Similarly, we have $\langle\mathbb{E}(u) \pm \boldsymbol{x}(u), \mathbb{E}(u)\rangle=1$ and $\left\langle\mathbb{L}^{+}(u), \mathbb{L}^{-}(u)\right\rangle=-2$ and these lead to the concepts of $\Delta_{3}$-duality and $\Delta_{4}$-duality respectively.

For spacelike hypersurfaces embedded in one of the pseudo-spheres in the Minkowski space (i.e. surfaces whose tangent spaces at all points are spacelike), we need to consider only the above four $\Delta_{i}$-dualities, $i=1, \ldots, 4$. However, if we consider timelike hypersurfaces in $S_{1}^{n}$, (i.e. surfaces whose tangent spaces at all points are timelike) we need the concept of $\Delta_{5}$-duality below which is also a direct analogue to the spherical 
duality in the Euclidean space. To summarise, we have the following five Legendrian double fibrations.

(1) (a) $H^{n}(-1) \times S_{1}^{n} \supset \Delta_{1}=\{(v, w) \mid\langle v, w\rangle=0\}$,

(b) $\pi_{11}: \Delta_{1} \rightarrow H^{n}(-1), \quad \pi_{12}: \Delta_{1} \rightarrow S_{1}^{n}$,

(c) $\theta_{11}=\langle d v, w\rangle\left|\Delta_{1}, \theta_{12}=\langle v, d w\rangle\right| \Delta_{1}$.

(2) (a) $H^{n}(-1) \times L C^{*} \supset \Delta_{2}=\{(v, w) \mid\langle v, w\rangle=-1\}$,

(b) $\pi_{21}: \Delta_{2} \rightarrow H^{n}(-1), \pi_{22}: \Delta_{2} \rightarrow L C^{*}$,

(c) $\theta_{21}=\langle d v, w\rangle\left|\Delta_{2}, \theta_{22}=\langle v, d w\rangle\right| \Delta_{2}$.

(3) (a) $L C^{*} \times S_{1}^{n} \supset \Delta_{3}=\{(v, w) \mid\langle v, w\rangle=1\}$,

(b) $\pi_{31}: \Delta_{3} \rightarrow L C^{*}, \pi_{32}: \Delta_{3} \rightarrow S_{1}^{n}$

(c) $\theta_{31}=\langle d v, w\rangle\left|\Delta_{3}, \theta_{32}=\langle v, d w\rangle\right| \Delta_{3}$.

(4) (a) $L C^{*} \times L C^{*} \supset \Delta_{4}=\{(v, w) \mid\langle v, w\rangle=-2\}$,

(b) $\pi_{41}: \Delta_{4} \rightarrow L C^{*}, \pi_{42}: \Delta_{4} \rightarrow L C^{*}$,

(c) $\theta_{41}=\langle d v, w\rangle\left|\Delta_{4}, \theta_{42}=\langle v, d w\rangle\right| \Delta_{4}$.

(5) (a) $S_{1}^{n} \times S_{1}^{n} \supset \Delta_{5}=\{(v, w) \mid\langle v, w\rangle=0\}$,

(b) $\pi_{51}: \Delta_{5} \rightarrow S_{1}^{n}, \pi_{52}: \Delta_{5} \rightarrow S_{1}^{n}$,

(c) $\theta_{51}=\langle d v, w\rangle\left|\Delta_{5}, \theta_{52}=\langle v, d w\rangle\right| \Delta_{5}$.

Above, $\pi_{i 1}(v, w)=v$ and $\pi_{i 2}(v, w)=w$ for $i=1, \ldots, 5,\langle d v, w\rangle=-w_{0} d v_{0}+$ $\sum_{i=1}^{n} w_{i} d v_{i}$ and $\langle v, d w\rangle=-v_{0} d w_{0}+\sum_{i=1}^{n} v_{i} d w_{i}$. The 1 -forms $\theta_{i 1}^{-1}$ and $\theta_{i 2}^{-1}, i=1, \ldots, 5$, define the same tangent hyperplane field over $\Delta_{i}$ which is denoted by $K_{i}$.

We have the following duality theorem on the above spaces.

Theorem 6.1 ([13, 14, 25]) The pairs $\left(\Delta_{i}, K_{i}\right), i=1, \ldots, 5$, are contact manifolds and $\pi_{i 1}$ and $\pi_{i 2}$ are Legendrian fibrations.

We have the following general remarks, some of which follow from the discussion proceeding Theorem 6.1.

Remark 6.2 1. Given a Legendrian submanifold $\boldsymbol{i}: L \rightarrow \Delta_{i}, i=1, \ldots, 5$, Theorem 6.1 states that $\pi_{i 1}(\boldsymbol{i}(L))$ is the $\Delta_{i}$-dual of $\pi_{i 2}(\boldsymbol{i}(L))$ and vice-versa.

2. We have the following geometric properties for a Legendrian submanifold $L \subset$ $\Delta_{i}, i=1, \ldots, 5$. Take the case $i=1$. If $\pi_{11}(\boldsymbol{i}(L))$ is smooth at a point $\pi_{11}(\boldsymbol{i}(\boldsymbol{u}))$, then $\pi_{12}(\boldsymbol{i}(\boldsymbol{u}))$ is the normal vector to the hypersurface $\pi_{11}(\boldsymbol{i}(L)) \subset H_{+}^{n}(-1)$ at $\pi_{11}(\boldsymbol{i}(\boldsymbol{u}))$. Conversely, if $\pi_{12}(\boldsymbol{i}(L))$ is smooth at a point $\pi_{12}(\boldsymbol{i}(\boldsymbol{u}))$, then $\pi_{11}(\boldsymbol{i}(\boldsymbol{u}))$ is the normal vector to the hypersurface $\pi_{12}(i(L)) \subset S_{1}^{n}$. The same holds for the $\Delta_{i}$-dualities, $i=2, \ldots, 5$, where we take the normal to a hypersurface $M \subset L C^{*}$ at $p \in M$ as the direction given by the intersection of the normal plane to $T_{p} M$ in $\mathbb{R}_{1}^{n+1}$ with $T_{p} L C^{*}$.

3. Since the normal of a hypersurface in $H^{n}(-1)$ is always spacelike, we have no good duality relationship in $H^{n}(-1) \times H^{n}(-1)$. 
Acknowledgement. The results in this paper were established during the visit of FT to Hokkaido University in October 2007. The visit was supported by a Royal Society International Outgoing Short Visit grant 2007/R2. We would like to thank the Royal Society for their support.

\section{References}

[1] A. I. Arnol'd, Singularities of systems of rays. Russ. Math. Surv. 38 (1983), 87-176.

[2] V. I. Arnol'd, S. M. Gusein-Zade and A. N. Varchenko, Singularities of Differentiable Maps vol. I. Birkhäuser, 1986.

[3] J. W. Bruce, Generic geometry and duality. Singularities (Lille, 1991), 29-59, London Math. Soc. Lecture Note Ser. 201, Cambridge Univ. Press, Cambridge, 1994.

[4] J. W. Bruce, Generic geometry, transversality and projections. J. London Math. Soc. 49 (1994), 183-194.

[5] J. W. Bruce, Projections and reflections of generic surfaces in $\mathbb{R}^{3}$. Math. Scand. 54 (1984), 262-278.

[6] J. W. Bruce and P. J. Giblin, Outlines and their duals. Proc. London Math. Soc. 50 (1985), 552-570.

[7] J. W. Bruce, P. J. Giblin and F. Tari, Families of surfaces: height functions and projections to plane. Math. Scand. 82 (1998), 165-185.

[8] J. W. Bruce, P. J. Giblin and F. Tari, Families of surfaces: height functions, Gauss maps and duals. Real and complex singularities (São Carlos, 1994). Pitman Res. Notes Math. Ser. 333 (1995), 148-178.

[9] J. W. Bruce and M. C. Romero-Fuster, Duality and projections of curves and surfaces in 3-space. Quart. J. Math. Oxford Ser. 42 (1991), 433-441.

[10] R. L. Bryant, Surfaces of mean curvature one in hyperbolic space. in Théorie des variétés minimales et applications (Palaiseau, 1983-1984), Astérisque No. 154-155, (1987),12, 321-347.

[11] R. Cipolla and P. J. Giblin, Visual Motion of Curves and Surfaces. Cambridge University Press, 2000.

[12] M. Golubitsky and V. Guillemin, Stable mappings and their singularities. Graduate Texts in Mathematics, Vol. 14. Springer-Verlag, New York-Heidelberg, 1973.

[13] S. Izumiya, Timelike hypersurfaces in de Sitter space and Legendrian singularities. $J$. Math. Sciences. 144 (2007), 3789-3803. 
[14] S. Izumiya, Legendrian dualities and spacelike hypersurfaces in the lightcone. Preprint, 2005.

[15] S. Izumiya, D. Pei and M. C. Romero-Fuster, The horospherical geometry of surfaces in Hyperbolic 4-space. Israel J. Math. 154 (2006), 361-379.

[16] S. Izumiya, D. Pei, M. C. Romero-Fuster and M. Takahashi, Horospherical geometry of submanifolds in hyperbolic n-space. J. London Math. Soc. 71 (2005), 779-800.

[17] S. Izumiya, D. Pei, M. C. Romero-Fuster and M. Takahashi, On the horospherical ridges of submanifolds of codimension 2 in Hyperbolic $n$-space. Bull. Braz. Math. Soc. 35 (2004), 177-198.

[18] S. Izumiya, D. Pei and T. Sano, Horospherical surfaces of curves in Hyperbolic space. Publ. Math. Debrecen 64 (2004), 1-13.

[19] S. Izumiya, D-H. Pei and T. Sano, Singularities of hyperbolic Gauss maps. Proc. London Math. Soc. 86 (2003), 485-512.

[20] S. Izumiya, D-H. Pei, T. Sano and E. Torii, Evolutes of hyperbolic plane curves. Acta Mathmatica Sinica 20 (2004), 543-550.

[21] S. Izumiya, D-H. Pei and M. Takahashi, Singularities of evolutes of hypersurfaces in hyperbolic space. Proc. Edinburgh Math. Soc. 47 (2004), 131-153.

[22] S. Izumiya, D-H. Pei and M. Takahashi, Curves and surfaces in Hyperbolic space. Banach Center Publications 65, Geometric singularity theory (2004), 197-123.

[23] S. Izumiya and M. C. Romero-Fuster, The horospherical Gauss-Bonnet type theorem in hyperbolic space. J. Math. Soc. Japan 58 (2006), 965-984.

[24] S. Izumiya, K. Saji and M. Takahashi, Horospherical flat surfaces in hyperbolic 3-space. Preprint, 2007.

[25] S. Izumiya and M. Takahashi, Spacelike parallels and evolutes in Minkowski pseudospheres. J. Geometry and Physics 57 (2007), 1569-1600.

[26] S. Izumiya, M. Takahashi and F. Tari, Folding maps on spacelike and timelike surfaces and duality. Preprint, 2007.

[27] S. Izumiya and F. Tari, Projections of surfaces in the hyperbolic space to hyperhorospheres and hyperplanes. To appear in Revista Matemática Iberoamericana.

[28] B. Karliğa and M. Savas, Orthogonal projections to a $k$-plane in hyperbolic, de Sitter and spherical $n$-spaces. Preprint, 2007.

[29] J. J. Koenderink, Solid shape. MIT Press Series in Artificial Intelligence. MIT Press, Cambridge, MA, 1990. 
[30] J. J. Koenderink, What does the occluding contour tell us about solid shape? Perception 13 (1984), 321-330.

[31] J. A. Montaldi, On generic composites of maps. Bull. London Math. Soc. 23 (1991), $81-85$.

[32] J. H. Rieger, Families of maps from the plane to the plane. J. London Math. Soc. 36 (1987), 351-369.

[33] J. H. Rieger, Versal topological stratification and the bifurcation geometry of map-germs of the plane. Math. Proc. Cambridge Philos. Soc. 107 (1990), 127-147.

[34] O. P. Shcherbak, Projectively dual space curves and Legendre singularities. Sel. Math. Sov 5 (1986), 391-421.

[35] V. M. Zakalyukin, Lagrangian and Legendre singularities. Funkcional. Anal. i Priložen. 10 (1976), 26-36.

S. Izumiya, Department of Mathematics, Hokkaido University, Sapporo 060-0810, Japan e-mail: izumiya@math.sci.hokudai.ac.jp

F. Tari, Department of Mathematical Sciences, Durham University, Science Laboratories, South Road, Durham DH1 3LE, UK

e-mail: farid.tari@durham.ac.uk 